\title{
III. Local Galactic Structure
}

\section{A. OPTICAL STUDIES}

This section contains work dealing with the Milky way characteristics in the solar neighbourhood and supported by observations with wavelengths shorter than $1 \mathrm{~mm}$. Thus, results are collected from gamma-ray, X-ray, UV, optical and IR observations in the different subsections. These are (1) the volume closest to the sun, (2) more distant objects at low galactic latitudes, and (3) objects at high galactic latitudes. Overall properties of the Galaxy and evolutionary effects are presented in Section IV.

1. The Volume Closest to the Sun

The nearby stars and the stellar luminosity function are discussed in detail in Section IID of this report.

Bahcall (37.155.014) used the distribution of $F$ stars to determine the amount of matter near the sun. Unobserved disk material is at least $50 \%$ of total disk material in the models considered.

Space densities for late-type giants have been determined by Ghosh and Tandon (32.155.063), and for Am stars by Ochsenbein (33.155.010). The mass spectrum in the solar neighbourhood was studied by Vereshchagin (34.155.062), and space densities for nearby stars were derived by Guseva et al. (33.155.019). Baggett (31.155.023) studied the stellar distribution in Gould's belt. Ishida and Mikami (31.155.014) have calculated the emissivity of the solar neighbourhood from density distributions of luminous stars.

Upgren and Chabotte (37.118.001) have examined about $200 \mathrm{~K}$ and $\mathrm{M}$ dwarfs with accurate parallaxes, proper motions and radial velocities. They find that the frequency of wide pairs can be accounted for by random space motions. Mezzetti et al. (33.155.102) point out the importance of taking unresolved binaries into account when deriving luminosity functions.

UV radiation from the sky back-ground was observed by Zvereva et a1. (32.157. 012) and compared to interstellar radiation at other wavelengths. Paresce ( 34.155. 072) shows that the state of the interstellar matter near the sun is consistent with a stellar wind or a supernova shock originating in the Sco-Cen association.

\section{Objects at Low Galactic Latitudes}

The effect of interstellar dust on observations of galaxies has been reviewed by de Vaucouleurs and Buta $(34.155 .002)$ who derive a csc-b-law. Nazarov and Khodyachikh (33.155.065) study the galactic extinction law from the apparent distribution of quasars.

In a series of papers $(32.155 .026,32.157 .011,33.155 .005,33.155 .006$, Winkler et al., 1984), the Bochum group present their photographic surface photometry of the Milky Way with a super-wide-angle camera. Reynolds (33.155.109) has mapped the Ho-emission-line profiles along the galactic equator. Feitzinger and Stüwe (1984) have catalogued dark nebulae and globules in longitudes $1=240^{\circ}-360^{\circ}$.

Optical observations of interest for galactic structure work include UBV $\beta$ photometry of luminous stars in the region $1=335^{\circ}-6^{\circ}$ (Dachs et a1., 32.113.031) and 8 -colour, Wing-system photometry of $M$ supergiants with $1=228^{\circ}-304^{\circ}$ (MacConnel1, Michigan State University). MacConnell also reports of a low-dispersion infrared 
objective-prism survey of $1=210^{\circ}-30^{\circ}, b= \pm 6^{\circ}$. Vega (31.113.092) searched for $\mathrm{H} \alpha$-emission-line objects in Vela; Bassino et a1. (32.113.043) studied faint $\mathrm{OB}$ stars in the Southern Milky Way; and Rabolli (33.113.058) made a UBV photometry of early-type stars near the HII region RCW 99. The spiral structure of the Sagittarius arm at $1=305^{\circ}$ was studied with radio, infrared and optical techniques by Danks et al. (37.155.023).

The Basel group has investigated luminosity and density functions based on RGU photometry in several Milky Way regions: $1=290^{\circ}, b=-0.3$ (Becker, Hassan, 31.155.007); a Centaurus field (Spaenhauer and Fang, 31.155.008); $1=993, b=12 \% 1$ (Becker and Fang, 31.155.048); $1=277 \% 0, \mathrm{~b}=0.8$ (Becker and Marsoglu, 32.113. 013); $1=39.7, b=-8.8$ (Fenkart et al., 33.113.019); towards Cassiopeia (Fenkart and Kandemir, 34.113.035); in the anticentre direction (in preparation). Two more parts (VIII, 1982, and IX, 1983) of the Basel photometric catalogue of stars in selected areas and other fields have also appeared.

Optical spiral structure at $1=30^{\circ}-70^{\circ}$ was discussed by Forbes $(33.155 .051$, 37.155.051). Neckel and Klare (32.155.055) discussed spiral structure out to $3-4$ $\mathrm{kpc}$ from the sun. The space distribution of stars in the direction of NGC 6823 was studied by Kuznetsov and Metreveli and space densities around the direction $1=$ $298^{\circ}, b=+1^{\circ}$ were derived by McCuskey (34.155.003). Burns et al. (37.155.012) studied the distribution of $\mathrm{B} 5-\mathrm{A} 5$ stars at $1=253^{\circ}, \mathrm{b}=-7^{\circ}$. Loden (Uppsala) has preliminarily concluded his study of galactic fine structure, particularly analysing the high clustering frequency among stars hotter than $F$ stars.

During the triennium, much attention has been given to galactic structure as observed in the infrared. In the near-infrared, the SRC atlas of the galactic plane was presented by Hartley and Dawe $(32,156.003)$. Sources were counted in the galactic plane by Kawara et a1. (33.155.123). There is also an objective-prism survey (Fuenmayor, 32.155.023) towards the galactic centre and the anticentre.

Presenting observations in the near-infrared from a rocket-borne telescope, Noguchi et a1. (31.156.009) conclude that most of the sources are late-type stars. The $2.4 \mu \mathrm{m}$ radiation enhancement at $1=355^{\circ}, \mathrm{b}=-1^{\circ}$ is related to newly detected M stars by Hamajima et a1. $(31,156,010)$, who conclude that extinction is low in that direction. Grasdalen et al. (34.155.065) used the AFGL infrared sky survey to study statistical properties of the stellar sources. Ananth and Nagaraja (32.156. 005) find that high fluxes at $2.2 \mu \mathrm{m}$ coincide with areas of strong correlation between far-infrared radiation and $\mathrm{CO}$ emission, indicating active star formation. Eaton et al. (37.155.060) observed stars at $2.2 \mu \mathrm{m}$; counts at different longitudes define an exponential disk.

Sources of far-infrared radiation have been studied by Jaffe et al. (31.156. 001 ) at $1=10^{\circ}-16^{\circ}$, and by Price et a1. (31.156.003) in the direction of Cygnus $x$. By utilising lunar-occultation methods, Stacey et a1. (33.155.111) were able to directly estimate fine-structure emission at $157 \mu \mathrm{m}$, i.e. [CII], from galactic longitude $1=8^{\circ}$. Bussoletti et al. (31.156.002) observed diffuse, far-infrared emission from the galactic plane at longitudes $233^{\circ}, 230^{\circ}, 222^{\circ}$ and $110^{\circ}$, and concluded that it originates in dust of around $10 \mathrm{~K}$.

The $\gamma$-radiation from the galactic anticentre was observed by Graser and Schönfelder (32.157.013), and interpreted as originating partly in the Crab Nebula. The diffuse $\gamma$-radiation between $1=100^{\circ}$ and $1=200^{\circ}$ is, according to Schlosser and Feitzinger (33.155.021), due to the central shingle of the local spiral arm. 


\section{Objects at High Galactic Latitudes}

Becker and Karaali (33.155.068) investigated a field near NGC 7006 using RGU photometry and determined density functions for both populations. The Basel halo program also includes several papers by Fenkart and collaborators (33.113.027, 34 . 113.036, and Astron. Astrophys. (in preparation)). Staller et a1. (31.155.003) found a large number of red objects near the South galactic pole and discussed the M-dwarf distribution. Trefzger et al. (1984) used Walraven photometry to show a metallicity gradient for stars in SA 141 at the South galactic pole. The density function of faint stars towards the North galactic pole was derived by Yoshii ( 32 . 155.034). Early-type stars near IC 4665 were investigated by Paparó and Balázs ( 33 . 155.124). Kron, Cudworth, and Rybski (Yerkes) are beginning a programme of photometry, spectroscopy and proper motions for complete samples of faint stars in about twenty fields at intermediate latitudes. Tobin $(37.113 .034,1984)$ has continued the studies by Tobin and Kaufmann (37.114.030) of high and intermediate-latitude $B$ stars; uvby $\beta$ photometry shows that some of these are more than $500 \mathrm{pc}$ from the galactic plane.

The globular cluster system was studied by Frenk and White $(31.155 .001)$, who concluded that the sun's distance to the galactic centre is $\mathrm{R}_{\mathrm{O}}=6.8 \pm 0.8 \mathrm{kpc}$.

\section{References}

Feitzinger, J.V., Stüwe, J.A.: 1984, Astron. Astrophys. Suppl. (in preparation). Tobin, W.: 1984, Astron. Astrophys. Suppl. (in press). Trefzger, Ch.F.: 1984, IAU Symposium No. 106 (in press). Winkler, Chr., Schmidt-Kaler, Th., Schlosser, W.: 1984, Astron. Astrophys. Suppl. (submitted).

\section{B. RADIO STUDIES}

Several regional surveys of southern HI made with the Argentine 100-m telescope were published $(30.155 .031)$. HI and other data were used by Dolidze (30.155. 044) to study the local distribution of gas and star forming regions. The Perseus arm region was the subject of an analogous study by Gerasimenko (34.155.139). Vallée (34.155.005) used rotation measure data from extragalactic sources in the direction of the Perseus arm to determine the magnetic field structure there and to constrain gravitational collapse theories of magnetic compression. HI in the direction of the Puppis window was surveyed by Stacy and Jackson (32.131.277) and used to study the turbulent characteristics of the interstellar medium. HI related to Gould's Belt was studied by Pöppel and Olano (32.155.024, 33.155.030). Salter (34. 131.014) reviewed radio and other observations pertaining to Loop I, the North Polar Spur, and considered the influence which this structure might have on the local medium.

Several regional studies were carried out in the radio continuum. Kononov and Pyatunina (34.155.017) surveyed the galactic plane in the region of Mononceros at $\lambda 7.6 \mathrm{~cm}$. The extended component of the radio continuum at 408,820 , and $1420 \mathrm{MHz}$ from the Cassiopeia-Perseus region was analyzed by Kallas et al. (34.155.069). Kanbach $(34.155 .020)$ reviewed the nonthermal radio emission from the local $(<2 \mathrm{kpc})$ region of the Galaxy and separated the emissivity into thin- and thick-disk components.

She11-1ike structures in the interstellar medium received much attention during the triennium. Hu $(30.155 .006)$ searched for HI shells at $|\mathrm{b}|>10^{\circ}$, using filtering in velocity to enhance the shell features; she found correlations of the HI shells with radio continuum loops. Gosachinskij $(31.132 .048)$ saw little correlation 